\title{
Gated SPECT left ventricular shape and prediction of super responders to cardiac resynchronization therapy: Not so easy as it (LV) looks
}

\author{
Mahmoud Altawil, MD, ${ }^{\mathrm{a}}$ Joshua Greenberg, $\mathrm{MD},{ }^{\mathrm{a}}$ and \\ Karthik Ananthasubramaniam, MD, FACC, FASNC ${ }^{a}$ \\ a Heart and Vascular Institute, Henry Ford West Bloomfield Hospital, West Bloomfield, MI
}

Received Jan 13, 2021; accepted Jan 14, 2021

doi: $10.1007 / \mathrm{s} 12350-021-02548-3$

\section{See related article, pp. 1537-1546}

Cardiac resynchronization therapy (CRT) remains a mainstay of treatment for patients with systolic heart failure and evidence of electrical dyssynchrony. In multiple large-scale clinic trials, patients with severely reduced left ventricular ejection fraction (LVEF) and wide QRS complex with left bundle branch block morphology show consistent and significant improvements after CRT including improvements in morbidity and mortality. ${ }^{1}$

Mechanistically, electrical dyssynchrony is thought to result in inter and intra-ventricular mechanical dyssynchrony and resultant impairments in left ventricular systolic function. Traditionally, the presence of a wide QRS left bundle branch block pattern remained the most important baseline characteristic predicting CRT response. $^{2}$ In fact, the current combined consensus guidelines of the American Heart Association/American College of Cardiology/Heart Rhythm Society recognize CRT as a class 1 indication for patients with LVEF < $35 \%$, sinus rhythm, New York Heart Association (NYHA) functional class $2 / 3$ or ambulatory class 4 heart failure and QRS duration $>150 \mathrm{~ms}$. A class 2A

\footnotetext{
Funding None

Mahmoud Altawil and Joshua Greenberg contributed equally to this manuscript.

Reprint requests: Karthik Ananthasubramaniam, MD, FACC, FASNC, Heart and Vascular Institute, Henry Ford West Bloomfield Hospital, 6777 West Maple, West Bloomfield, MI 48322; kananth1@hfhs.org J Nucl Cardiol 2022;29:1547-51.

$1071-3581 / \$ 34.00$

Copyright (C) 2021 American Society of Nuclear Cardiology.
}

indication is for heart failure patients with a QRS duration between 120 and 149 msec or those with non left bundle branch block QRS duration of $150 \mathrm{msec}^{3}$ Despite this, 20-40\% of these patients with electrical dyssynchrony as evidenced by wide QRS, do not demonstrate significant cardiac functional improvement after CRT. ${ }^{4}$ In this way, the reliable identification of socalled "non-responders" to CRT remains a significant challenge. To date, no single clinical, electrocardiographic, or echocardiographic parameters can sub-select CRT responders prior to device implantation. ${ }^{5}$

To complicate things further, electrical dyssynchrony may not always correlate with mechanical dyssynchrony or predict CRT response. ${ }^{6}$ In this manner, direct assessment of mechanical dyssynchrony and baseline structural dysfunction remain appealing targets to predict CRT response.

Clinical and echocardiographic evidence for CRT response also may be discordant. CRT trials have utilized many factors to establish CRT response including NYHA class improvements, heart failure morbidity/ hospitalizations, quality of life parameters, cardiac and overall mortality, echocardiographic LVEF changes, and decreases in left ventricular volumes/volume indices.

Studies have also demonstrated that there is a poor agreement regarding variables/cutoffs to define response and super response to CRT in general. ${ }^{7}$ The concept of super response or super-responder to CRT is not standardized and multiple studies have used various definitions of a " super responder', to CRT. 8,9 Furthermore, super responders, although showing a more 
robust response to CRT by certain criteria, are no different in their dependence on continued CRT despite improvement in LV function and negative remodeling of LV. Recent studies have shown that once CRT is turned off in super responders, LV adverse remodeling and drop in EF still recur with adverse outcomes, suggesting that super response by no means is a ticket to safety and that CRT may be a lifelong requirement once instituted. Similarly, implantable cardioverter-defibrillator discharges continue to occur in super responders although less than in "responders. ${ }^{10}$

The relationship between LV shape and adverse remodeling in response to heart failure is one of the most widely studied parameters for prognosis. ${ }^{11}$ Although in early stages the remodeling is a Frank Starling law based response to maintain cardiac output and $\mathrm{EF}$, over the course of heart failure there is a progressive loss of ellipsoidal shape of LV towards a spherical shape causing progressive dysfunction and increased wall stress. With CRT, there is a decrease in LV size and improvement in LVEF observed within the first few months of the implant, which continues over 6-12 months after implant. ${ }^{12}$ Thus, an LV that is less deformed to begin with, would naturally have a greater capability of negative remodeling post-CRT regardless of etiology. Disappointingly, echocardiographic parameters of dyssynchrony and LV shape change show large variability and limited sensitivity and specificity to predict CRT response. ${ }^{13}$

Recently, gated single-photon emission computed tomography (gSPECT) has emerged as a promising imaging modality to assess myocardial dyssynchrony. The concept of using gSPECT to evaluate LV dyssynchrony has been pioneered by the Emory group ${ }^{14}$ and the reader is referred to detailed reviews published regarding phase analysis and its potential role in identifying and guiding CRT lead placement and potential response or lack thereof to CRT. ${ }^{15}$

gSPECT is an established modality not only for diagnosis and prognosis of coronary disease but also by its ability to provide robust information on EF, enddiastolic and end-systolic volumes, which are established parameters in clinical practice. However, 3dimensional LV shape change assessment using gSPECT data is less familiar with regards to implications in clinical practice. LV shape index and its prognostic value in heart failure derived from gSPECT was described 14 years ago in this journal by Abidov et al. ${ }^{16}$ Recent studies have explored various aspects of LV shape index and its relationship to adverse outcomes. ${ }^{17}$

As a prelude to discussing the study by Zhou et al. a brief recap of VISION-CRT study would be pertinent. The VISION-CRT investigators assessed the role of
gSPECT myocardial perfusion imaging (MPI) to predict clinical outcomes in patients undergoing CRT. This prospective, non-randomized study evaluated 195 patients with NYHA class $2 / 3$ or ambulatory class 4 heart failure on optimal medical therapy for at least 3 months. Other inclusion criteria included an LVEF < $35 \%$, QRS $>120 \mathrm{~ms}$ with left bundle branch block morphology, and the presence of sinus rhythm to allow SPECT gating.

The investigators performed gSPECT MPI at baseline and 6 months after CRT placement and assessed LV dyssynchrony using the validated measure of gSPECT MPI phase histogram standard deviation (PSD). ${ }^{18}$ Software analysis of gSPECT MPI was automated and performed in a central core laboratory utilizing the Emory Cardiac Toolbox. Primary outcomes included improvement in NYHA functional class by 1 , LVEF improvement by $>5 \%$, Minnesota Living with Heart Failure Questionnaire score increase of 5 points, and left ventricular end-systolic volume decrease by $>$ $15 \%$. In VISION-CRT, 179/198 patients completed follow-up with at least one primary outcome occurring in $84.9 \%(\mathrm{n}=152)$ participants. Notably, baseline PSD was not associated with the primary outcome on both univariate (odds ratio [OR] $0.7,95 \%$ CI $0.33-1.5$ ) or multivariate analysis (OR $0.66,95 \%$ CI $0.25-1.76$ ).

Improvement in LV PSD at 6 months from baseline was associated with a small but significant improvement in the primary outcome (OR 1.04, 95\% CI 1.01-1.07) per 1-degree decrease in LV PSD.

Overall the study showed the limited predictive utility of CRT response utilizing gSPECT MPI PSD as a surrogate for mechanical dyssynchrony. ${ }^{18}$

Utilizing the data set from the Vision-CRT, Zhou et al in this issue of the journal evaluated the potential for the gSPECT defined LV shape parameters (left ventricular end-systolic eccentricity [ESE] and left ventricular end-diastolic eccentricity [EDE]) to predict "super-responders" enrolled in the VISION-CRT trial. ${ }^{18}$ CRT "super response" was defined as an increase in LVEF $\geq 15 \%$. The investigators hypothesized that increased LV eccentricity at baseline would predict CRT response. Myocardial eccentricity was derived from the below equation.

$$
\text { Eccentricity }=\sqrt{1-\frac{x \times y}{z^{2}}}
$$

Using the above equation and assuming the minor axes $(x=y)$ as the major axis (z) decreases, eccentricity increases in turn. Eccentricity values closer to 1 represent spherical geometry and values closer to 0 represent ellipsoid geometry. Zhou et al noted significant differences in ESE and EDE (ESE: $0.6 \pm 0.1$ vs $0.5 \pm 0.2, P=$ 
0.005 ; EDE: $0.6 \pm 0.2$ vs $0.5 \pm 0.2, P=0.037$ ) between CRT super responders and non-super responders. Furthermore, ESE remained an independent predictor of CRT super responders in univariate (OR 12.59, 95\% CI 1.56-101.35, $P=0.017$ ) and multivariate analyses (OR 35.71, 95\% CI 1.66-766.03, $P=0.006)$. ESE also had incremental value over significant clinical and nuclear imaging variables, including angiotensin-converting enzyme inhibitors or angiotensin II receptor blocker use, coronary artery disease, myocardial infarction, LVEF, and scar burden in predicting CRT super responders (area under the curve 0.82 vs 0.80 , sensitivity 0.68 vs 0.65 , and specificity 0.82 vs 0.78 ). They concluded that LV shape parameters from gSPECT, specifically ESE, provide incremental value to predict super response to CRT over existing clinical and nuclear imaging variables.

The investigators did note the following limitations of the study: restriction to a post hoc analysis of the nonrandomized VISION-CRT trial data, limited 6-month follow-up, small patient numbers and absence of clinical variables including heart rate during image acquisition.

The study does have several other notable limitations not addressed by the authors. Specifically, while ESE was significantly associated with super response on univariate and multivariate analysis, the confidence intervals are wide (95\% CI 1.56-101.35 and 95\% CI 1.66-766.03, respectively).

Furthermore, while the differences in ESE/EDE between non-responders and super responders are significant, the absolute differences are minimal (ESE: 0.6 \pm 0.1 vs. $0.5 \pm 0.2, P=0.005$; EDE: $0.6 \pm 0.2$ vs. $0.5 \pm$ $0.2, P=0.037)$. Small absolute changes and the wide confidence intervals do raise the questions of generalizability and reproducibility in the clinical setting outside of core laboratory measurements. It should be noted though that a prior study from 2009 did show that the intra-observer and inter-observer reproducibility for the eccentricity indices were highly correlated (ICCs: 0.99, 95\% CI 0.98-0.99 and ICCs: 0.98, 95\% CI 0.970.99, respectively). ${ }^{19}$ Also, while receiver operator curves did show improvement in modeling CRT super response with the addition of ESE, the absolute improvement was marginal (area under the curve 0.82 vs 0.80 , sensitivity 0.68 vs 0.65 , specificity 0.82 vs 0.78 ) and of unclear clinical relevance.

Also while ESE was predictive of generalized CRT response, defined as $\mathrm{LVEF}>5 \%$ on univariate analysis $(0.6 \pm 0.1$ vs $0.5 \pm 0.2, P<0.001)$, ESE was not significantly predictive of CRT response on multivariate analysis. It was only upon limiting the analysis to super responders that ESE became significant on both univariate and multivariate analysis. While the authors utilized a definition of CRT response as LVEF improvement of $5 \%$ and super response LVEF $>15 \%$, there are in fact no established definitions for CRT response or super response. As the study definition of "super responders", hinges on LVEF criteria, this merits discussion.

Using an EF cutoff alone to predict response or super response may miss identifying individuals who have favorable LV remodeling (negative remodeling) without necessarily the expected EF change. As discussed before LV negative remodeling has been felt to be the most important predictor of long-term favorable outcome post-CRT. ${ }^{9,20}$ Using the authors' definition of super responders, the percentage of super responders is high $(44 \%)$ when compared to other contemporary studies. ${ }^{7}$ Furthermore studies evaluating CRT response typically utilized echocardiographic analysis of LVEF and LV volumes and not SPECT. The VISION-CRT investigators did utilize transthoracic echocardiography at baseline and further analysis of LVEF and LV volumes was performed by SPECT. Interestingly, only a minority of patients submitted pre- and post-echocardiography images to the processing core laboratory. Among those, the correlations of ESV/ESE/EDV/EDE between echocardiography and SPECT were low. Prior studies have also noted significant discordance in the estimate of LVEF between SPECT and utilize transthoracic echocardiography particularly in patients with reduced systolic function. ${ }^{21}$

Other potential confounding variables not included in the VISION data set or the analysis by Zhou et al include etiologies of congestive heart failure (non-ischemic vs ischemic), duration of heart failure symptoms, left atrial size, blood pressure, presence of significant valvular pathology, paroxysmal arrhythmia burden, paced QRS duration, absolute CRT pacing, and effective CRT pacing. Additionally, CRT programming parameters/settings were not standardized. There is a multitude of CRT parameters/programming variations that could confound CRT response including upper and lower rate limits, rate-adaptive pacing, pacing polarity, fixed vs dynamic LV offsets, multi-point pacing, fixed vs dynamic atrioventricular delays, left ventricular only/ adaptive LV pacing and the utilization of acute heart failure diagnostic algorithms.

Regardless, Zhuo et al have eloquently utilized gSPECT data from the previous VISION-CRT data set to describe a SPECT-derived measure of eccentricity, ESE, which could provide incremental value in the determination of CRT super responders. While their post hoc analysis has inherent limitations and the clinical utility of ESE in predicting CRT response remains uncertain, their preliminary conclusions are intriguing. gSPECT dyssynchrony and LV shape change information remains a largely untapped area for integrating into 
CRT planning and predicting response. Of all imaging modalities studied in CRT so far, gSPECT information appears to be the most reproducible and easy to obtain with early evidence to help guide therapy with phase analysis. Thus along with phase analysis data potentially incorporating other measures such as LVEF and LV shape change analysis could be of value in discriminating those who have an excellent response to CRT if confirmed in larger studies. Although the editorialists believe that no single imaging modality by itself can predict CRT response or super response, it is time to see if gSPECT fills part of the void and integrates with multiple other complex factors as outlined above for predicting CRT response and possibly identify super responders. The study by Zhou et al is another step forward to encourage prospective randomized multicenter evaluation of gSPECT parameters with clinical and CRT settings to best predict response.

\section{Acknowledgements}

The authors thank Stephanie Stebens from Sladen Library, Henry Ford Hospital for her expert manuscript preparation and assistance.

\section{Disclosures}

Dr Ananthasubramaniam receives research grants from Astellas Pharma and Alnylam Pharmaceuticals and is on advisory panel of Alnylam Pharmaceuticals. Drs Altawil and Greenberg declare that they have no relevant conflicts of interest

\section{References}

1. Bristow MR, Saxon LA, Boehmer J, Krueger S, Kass DA, De Marco T, Comparison of Medical Therapy, Pacing, and Defibrillation in Heart Failure (COMPANION) Investigators et al (2004) Cardiac-resynchronization therapy with or without an implantable defibrillator in advanced chronic heart failure. $\mathrm{N}$ Engl J Med 350:2140-50

2. Leclercq C, Kass DA (2002) Retiming the failing heart: Principles and current clinical status of cardiac resyncrhonization. J Am Coll Cardiol 39:194-01

3. Yancy CW, Jessup M, Bozkut B, Butler J, Casey DE Jr, Drazner $\mathrm{MH}$, American College of Cardiology Foundation, American Heart Association Task Force on Practice Guidelines et al (2013) ACCF/AHA guideline for the management of heart failure: A report of the American College of Cardiology Foundation/American Heart Association Task Force on Practice Guidelines. J Am Coll Cardiol 2013(62):e147-39

4. Hsing JM, Selzman KA, Leclercq C, Pires LA, McLaughlin MG, McRae SE et al (2011) Paced left ventricular QRS width and ECG parameters predict outcomes after cardiac resynchronization therapy: PROSPECT-ECG substudy. Circ Arrhythm Electrophysiol 4:851-57
5. Steffel J, Leclercq C (2014) Mechanical dyssynchrony in CRT: Still searching for the Holy Grail. Eur Heart J 35:13-15

6. Sillanmaki S, Lipponen JA, Tarvainen MP, Laitinen T, Hedman M, Hedman A et al (2019) Relationships between electrical and mechanical dyssynchrony in patients with left bundle branch block and healthy controls. J Nucl Cardiol 26:1228--39

7. Steffel J, Ruschitzka F (2014) Superresponse to cardiac resynchronization therapy. Circulation 130:87-90

8. Steffel J, Milosevic G, Hürlimann A, Krasniqi N, Namdar M, Ruschitzka F et al (2011) Characteristics and long-term outcome of echocardiographic super-responders to cardiac resynchronisation therapy: "Real world" experience from a single tertiary care centre. Heart 97:1668-74

9. Ypenburg C, van Bommel RJ, Borleffs CJ, Bleeker GB, Boersma E, Schalij MJ et al (2009) Long-term prognosis after cardiac resynchronization therapy is related to the extsnt of left ventricular reverse remodeling at midterm follow-up. J Am Coll Cardiol 53:483-90

10. Killu AM, Mazo A, Grupper A, Madhavan M, Webster T, Brooke KL et al (2018) Super-response to cardiac resynchronization therapy reduces appropriate implantable cardioverterr defibrillator therapy. Europace 20:1303-11

11. Bluemke DA, Kronmal RA, Lima JA, Liu K, Olson J, Burke GL et al (2008) The relationship of left ventricular mass and geometry to incident cardiovascular events: The MESA (Multi-Ethnic Study of Atherosclerosis) study. J Am Coll Cardiol 52:2148-55

12. St John Sutton MG, Plappert T, Abraham WT, Smith AL, DeLurgio DB, Leon AR, Multicenter InSync Randomized Clinical Evaluation (MIRACLE) Study Group et al (2003) Effect of cardiac resynchronization therapy on left ventricular size and function in chronic heart failure. Circulation 107:1985-90

13. Chung ES, Leon AR, Tavazzi L, Sun JP, Nihoyannopoulos P, Merlino J et al (2008) Results of the predictors of response to CRT (Prospect) trial. Circulation 117(2608):16

14. Chen J, Garcia EV, Folks RD, Cooke CD, Faber TL, Tauxe EL et al (2005) Onset of left ventricular mechanical contraction as determined by phase analysis of ECG-gated myocardial perfusion SPECT imaging: Development of a diagnostic tool for assessment of cardiac mechanical dyssynchrony. J Nucl Cardiol 12:687-95

15. Soman P, Chen J (2014) Left ventricular dyssynchrony assessment using myocardial single-photon emission CT. Semin Nucl Med 44:314-19

16. Abidov A, Slomka PJ, Nishina H, Hayes SW, Kang X, Yoda S et al (2006) Left ventricular shape index assessed by gated stress myocardial perfusion SPECT: Initial description of a new variable. J Nucl Cardiol 13:652-59

17. Gaudieri V, Nappi C, Acampa W, Zampella E, Assante R, Mannarino $T$ et al (2019) Added prognostic value of left ventricular shape by gated SPECT imaging in patients with suspected coronary artery disease and normal myocardial perfusion. J Nucl Cardiol 26:1148-56

18. Peix A, Karthikeyan G, Massardo T, Kalaivani M, Patel C, Pabon LM et al (2019) Value of intraventricular dyssynchrony assessment by gated-SPECT myocardial perfusion imaging in the management of heart failure patients undergoing cardiac resynchronization therapy (VISION-CRT). J Nucl Cardiol. https://doi. org/10.1007/s12350-018-01589-5

19. Gimelli A, Liga R, Clemente A, Marras G, Kusch A, Marzullo P (2020) Left ventricular eccentricity index measured with SPECT myocardial perfusion imaging: An additional parameter of adverse cardiac remodeling. J Nucl Cardiol 27:71-79

20. Yu CM, Bleeker GB, Fung JW, Schalij MJ, Zhang Q, van der Wall EE et al (2005) Left ventricular reverse remodeling but not clinical 
improvement predicts long-term survival after cardiac resynchronization therapy. Circulation 112:1580-86

21. Rawala M, M. A. (2019) Discordance in LVEF between nuclear SPECT and echocardiography in patients with moderate reduction in LVEF. J Diagn Med Sonogr 35(4):290-95
Publisher's Note Springer Nature remains neutral with regard to jurisdictional claims in published maps and institutional affiliations. 